\title{
Fatigue life prediction under mixed-mode loading using equivalent stress intensity factor models
}

\author{
S. Sajith ${ }^{1, *}$, K.S.R. K. Murthy ${ }^{2}$, and P.S. Robi ${ }^{2}$ \\ ${ }^{1}$ Research Scholar, Department of Mechanical Engineering, Indian Institute of Technology Guwahati, Guwahati - 781039, India. \\ ${ }^{2}$ Professor, Department of Mechanical Engineering, Indian Institute of Technology Guwahati, Guwahati - 781039, India.
}

\begin{abstract}
Damage tolerance principles are widely used to assess the structural integrity and failure of engineering components. The advances in numerical simulation techniques facilitate the prediction of fatigue life of engineering component, which is essential in damage tolerance design. For the components under mixed mode (I/II) loading, the fatigue crack growth and life is predicted by using a modified form of Paris' law along with the equivalent stress intensity factor $\left(\Delta K_{e q}\right)$. Numerous $\Delta K_{e q}$ models are available for correlating the equivalent stress intensity factor range and the fatigue crack growth rate. The knowledge of proper $\Delta K_{e q}$ model is essential for the accurate fatigue life estimation. In this work, the authors numerically assess the performance of $\Delta K_{e q}$ models in mixed mode fatigue life prediction with the help of published experimental mixed mode data.
\end{abstract}

Keywords. Fatigue; mixed mode; crack growth; stress intensity factor; fatigue crack

\section{Introduction}

Engineering components and parts are generally assumed to be safe, but they contain randomly oriented microcracks, voids, inclusions and flaws, and that may grow under cyclic loading. For the cracks subjected to repeated or cyclic loading, fatigue crack growth analysis based on fracture mechanics principles plays an important role in damage tolerance assessments and in the evaluation of residual fatigue life. Structural components having complex geometries require the use of numerical simulation techniques for the fatigue crack growth (FCG) rate and fatigue life prediction. Numerical techniques such as boundary element and finite element methods are generally used for this purpose.

For the ease of analysis, most of the engineering components containing cracks are considered to be either under mode I or mode II loading and are assumed to be under static loading. Numerous crack growth laws are available for the fatigue life correlation for cracks subjected to only mode I loading. For cracks under opening mode/mode I loading, the power law proposed by Paris [1] has received wide acceptance. Under mixedmode (I/II) loading conditions, the crack propagation is in non-self-similar manner, i.e., the crack deviates from its original crack path and crack growth rate is significantly affected by the mixed mode loading condition. So in mixed mode loading, the crack path as well as the crack growth rate has to be determined. For cracks subjected to mixed mode loading, Tanaka [2] proposed the modified form of Paris law, a relationship between the fatigue crack growth rate and equivalent stress intensity factor (equivalent SIF) range, which is given by

$$
\frac{d a}{d N}=C\left(\Delta K_{e q}\right)^{m}
$$

where

$$
\Delta K_{e q}=f\left(\Delta K_{I}, \Delta K_{I I}\right)
$$

where $C$ and $m$ are material constants. Equation (1) represents the linear relation between on logarithmic scale. The material constants $C$ and $m$ can be obtained from mode I fatigue tests [3].

A large number of $\Delta K_{e q}$ models have been proposed for establishing a better relationship between equivalent SIF $\Delta K_{e q}$ and the crack growth rate $d a / d N$ under mixed mode I/II loading conditions. Accuracy of the numerically estimated fatigue cracks growth rate always depends on the selected $\Delta K_{e q}$ model. To the best of authors' knowledge, no work is available on comparison of predictability of different available expressions for the parameter $\Delta K_{e q}$ (for a given path prediction rule) in numerical prediction of crack length versus number of loading cycles against available experimental data despite the importance of simulated fatigue life in damage tolerance philosophy. Thus the present investigation attempts to assess the performances of

Corresponding author: s.sajith@iitg.ernet.in 
different models for $\Delta K_{e q}$ in numerical prediction of crack growth curves by comparing with the same published experimental data.

\section{Methodology and theoretical formulations}

\subsection{Numerical estimation of mixed mode fatigue life}

This section briefly describes the numerical procedure for simulation of mixed mode (I/II) fatigue crack growth. Fig.1 shows different steps to carryout fatigue crack growth numerical simulation under mixed mode loading. An incremental crack tip extension method is used in present study, in which the crack tip is advanced to a new tip position after each step. At each step, SIFs $K_{I}$ and $K_{I I}$ are calculated. At the end of each step, the fatigue crack growth direction, as well as fatigue life, is calculated using the SIFs. The new crack tip coordinates are being calculated and the crack is advanced to the new position. The crack step increments considered are relatively small (w:r:t the initial crack length), for obtaining a smooth crack path. The finite element numerical simulations were carried out using the crack propagation simulator FRANC2D, which is a finite element based crack propagation simulator. The necessary scripts for the post-processing were developed in MATLAB.

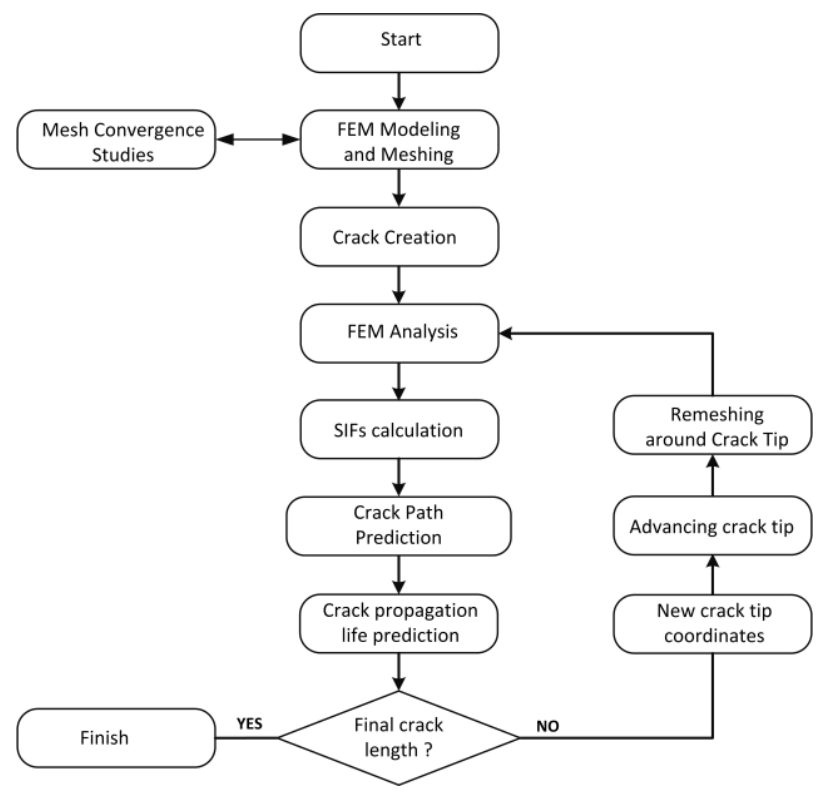

Fig. 1. Typical flow chart for mixed mode fatigue life estimation.

\subsubsection{Fatigue crack path prediction}

Accurate estimation of fatigue crack path is essesntial for the fatigue life pediction. Erdogan and Sih [4] proposed maximum tangential stress criterion (MTS), which is given as

$$
K_{I} \sin \theta_{c}+K_{I I}\left(3 \cos \theta_{c}-1\right)=0
$$

Solving the above equation for the propagation angle $\theta$

$$
\begin{aligned}
& \theta_{c}=2 \tan ^{-1}\left[\frac{1}{4} \frac{K_{I}}{K_{I I}}-\frac{1}{4} \sqrt{\left(\frac{K_{I}}{K_{I I}}\right)^{2}+8}\right] \text { for } K_{I I}>0 \\
& \theta_{c}=2 \tan ^{-1}\left[\frac{1}{4} \frac{K_{I}}{K_{I I}}+\frac{1}{4} \sqrt{\left(\frac{K_{I}}{K_{I I}}\right)^{2}+8}\right] \text { for } K_{I I}<0
\end{aligned}
$$

where positive $\theta_{c}$ is the crack growth direction from the initial crack axis in anticlockwise direction.

\subsubsection{Fatigue life prediction}

The fatigue crack propagation prediction using the equivalent SIF $\Delta K_{e q}$ is the most widely used approach for components under mode I/II cyclic loading. Using a modified form of Paris law, Tanaka [2] proposed a power law for the correlation of fatigue crack growth rate and equivalent $\operatorname{SIF} \Delta K_{e q}$ ( under mixed mode I/II), which is given as

$$
\frac{d a}{d N}=C\left(\Delta K_{e q}\right)^{m}
$$

From Eq. (5), for a crack increment, the fatigue life cycles can be determined as

$$
\int_{0}^{\Delta a} \frac{d a}{c\left(\Delta K_{e q}\right)^{m}}=\int_{0}^{\Delta N} d N=\Delta N
$$

Some of the commonly used $\Delta K_{e q}$ models along with the proposed authors are summarized in Table 1.

Table 1. List of $\Delta K_{e q}$ models along with their notations used in the present study.

\begin{tabular}{|c|c|}
\hline Notation & $\Delta K_{e q}$ models/expression \\
\hline $\begin{array}{c}\text { Tanaka_1 } \\
\text { [2] }\end{array}$ & $\Delta K_{e q}=\left(\Delta K_{I}^{2}+2 \Delta K_{I I}^{2}\right)^{1 / 2}$ \\
\hline $\begin{array}{c}\text { Tanaka_2 } \\
\text { [2] }\end{array}$ & $\Delta K_{e q}=\left(\Delta K_{I}^{4}+8 \Delta K_{I I}^{4}\right)^{1 / 4}$ \\
\hline Irwin [5] & $\Delta K_{e q}=\sqrt{\Delta K_{I}^{2}+\Delta K_{I I}^{2}}$ \\
\hline $\begin{array}{c}\text { Yan et al. } \\
\text { [6] }\end{array}$ & $\Delta K_{e q}=\frac{1}{2 \cos \frac{\theta}{2}\left[\Delta K_{I}(1+\cos \theta)-3 \Delta K_{I I} \sin \theta\right]}$ \\
\hline $\begin{array}{c}\text { Hussain } \\
\text { et al. [7] }\end{array}$ & $\Delta K_{e q}=\sqrt{\frac{4}{\left(3+\cos ^{2} \theta\right)^{2}}\left(\frac{1-\theta / \pi}{1+\theta / \pi}\right)^{\theta / \pi}\left[\begin{array}{l}\left(1+3 \cos ^{2} \theta\right) \Delta K_{I}^{2}+ \\
4 \sin 2 \theta \Delta K_{I} \Delta K_{I I}+ \\
\left(9-5 \cos ^{2} \theta\right) \Delta K_{I I}^{2}\end{array}\right.}$ \\
\hline $\begin{array}{c}\text { Richard } \\
\text { et al. [8] }\end{array}$ & $\Delta K_{e q}=\frac{\Delta K_{I}}{2}+\frac{1}{2} \sqrt{\Delta K_{I}^{2}+4\left(1.155 \Delta K_{I I}\right)^{2}}$ \\
\hline
\end{tabular}

The equivalent SIF models listed in Table 1 were developed to establish a linear relationship between the experimental $\frac{d a}{d N}$ and equivalent $\operatorname{SIF} \Delta K_{e q}$ on a 
logarithmic scale. A performance evaluation of the equivalent SIF models (in predicting the fatigue life) is carried out, and they are discussed in the following section.

\subsection{Performance assessment of Equivalent SIF models}

This section discusses the finite element simulations performed for the present investigation. The numerical simulation is based on the experimental studies using mixed mode I/II CTS specimen by Ma et al. [9] The geometry and boundary conditions of the mixed mode CTS specimen used for the present investigation is given in Fig. 2(a). The loading direction is making an angle of $60^{\circ}$ with crack axis. The experimental specimen made of steel is used by Ma et al. [9] in his experimental studies. For applying mixed mode loading using the uni-axial testing machine, Richard's loading device [10] is used. Fig. 2(b) shows the boundary conditions and equivalent loading used for the finite element simulations. The uniaxial load $P$ is related to the equivalent loads acting on different holes according to the following equations

$$
\begin{aligned}
& P_{1}=P_{6}=P(0.5 \cos \theta+(c / b) \sin \theta) \\
& P_{2}=P_{5}=P \sin \theta \\
& P_{3}=P_{4}=P(0.5 \cos \theta-(c / b) \sin \theta)
\end{aligned}
$$

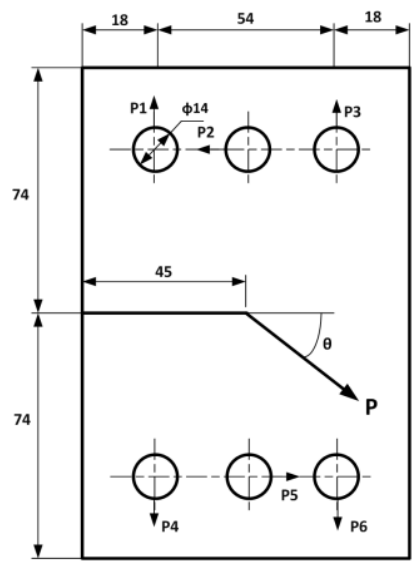

(a)

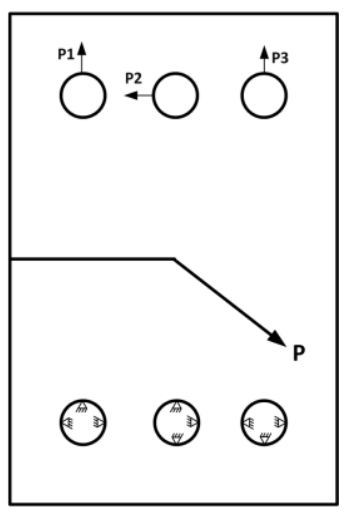

(b)
Fig. 2. (a) The dimensions of experimental CTS specimen used by $\mathrm{Ma}$ et al. [9] (b) Boundary conditions and the equivalent loading of the specimen used for numerical simulation.

The material used for the experiment, material properties and test conditions given by $\mathrm{Ma}$ et al. [9] along with material properties and other constants used for present analysis are given in table 2 . An incremental crack tip extension method is followed in the present numerical investigation, and the step crack increment $\Delta a=0.5 \mathrm{~mm}$ is used. For modeling the material response, plane stress condition is assumed.

Table 2. Material properties and test condition data for CTS specimen.

\begin{tabular}{|l|l|}
\hline $\begin{array}{l}\text { Material used for } \\
\text { experiment }\end{array}$ & Steel S460 \\
\hline Young's Modulus, $E$ & $200 \mathrm{GPa}$ \\
\hline Poisson's ratio, $v$ & 0.25 \\
\hline Plate thickness, $t$ & $6 \mathrm{~mm}$ \\
\hline Paris constant, $C$ & $7 \mathrm{E}-8((\mathrm{~m} / \mathrm{cycle}) /(\mathrm{MPa} \sqrt{\mathrm{m}}))^{\mathrm{m}}$ \\
\hline Paris exponent, $m$ & 2.1 \\
\hline Load $\Delta P$ & $9 \mathrm{kN}$ \\
\hline Load ratio $R$ & 0.5 \\
\hline Frequency $f$ & $25 \mathrm{~Hz}$ \\
\hline Initial $K_{I I} / K_{I}$ & 0.3 \\
\hline Yield strength & $460 \mathrm{MPa}$ \\
\hline Ultimate strength & $610 \mathrm{MPa}$ \\
\hline Crack increment $\Delta a$ & $0.5 \mathrm{~mm}$ \\
\hline Initial crack length $a_{i}$ & $45 \mathrm{~mm}$ \\
\hline Final crack length $a_{f}$ & $65 \mathrm{~mm}$ \\
\hline
\end{tabular}

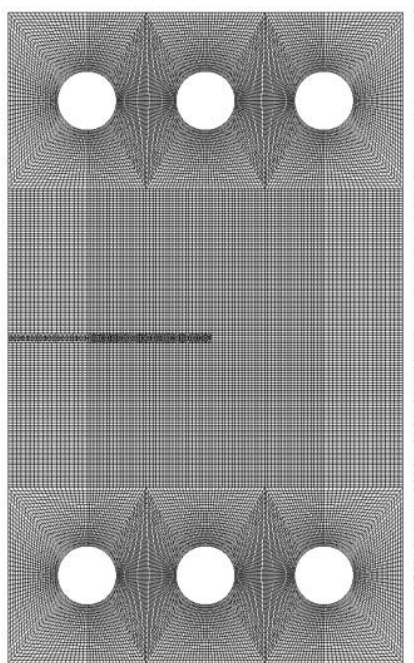

(a)

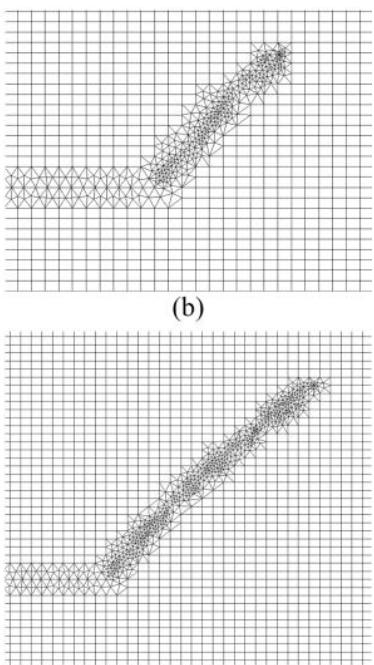

(c)
Fig. 3. (a) Initial mesh, (b) intermediate mesh and (c) final mesh used for finite element simulation.

The fatigue crack growth analysis from initial crack length $a_{0}=45 \mathrm{~mm}$ to final crack length $a_{f}=65 \mathrm{~mm}$ with 40 steps in carried out. Mixed mode SIFs $K_{I}$ and $K_{I I}$ are calculated at each step using displacement methods. The calculated mixed-mode stress intensity factors are utilized for the equivalent SIF calculation and for the crack propagation direction using MTS criterion. Using the crack kink direction and the constant crack increment, the new location of the crack tip is calculated, and the crack is advanced to the new position. Fig. 4 
shows the crack path obtained from the present numerical simulations. The experimental crack path was not available for the present problem considered. Fig. 5 shows experimental fatigue life curves by Ma et al. [9] and a six-degree polynomial fit on experimental data. It can be noted from the goodness of fit of Fig. 5 that the polynomial fit represents the experimental data accurately.

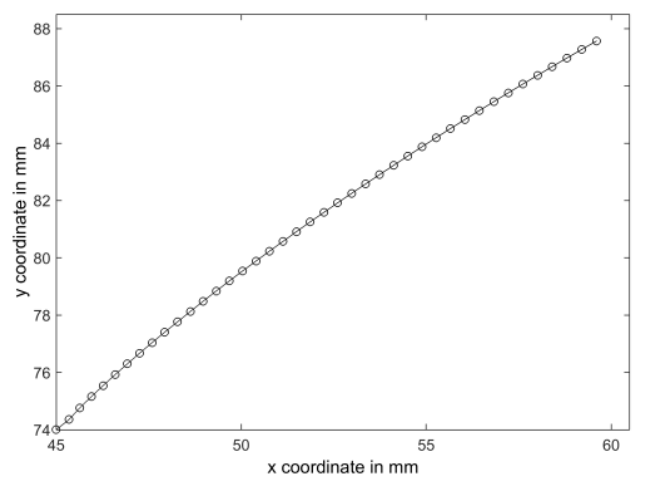

Fig. 4. The simulated crack path for CTS specimen of $60^{\circ}$ loading angle.

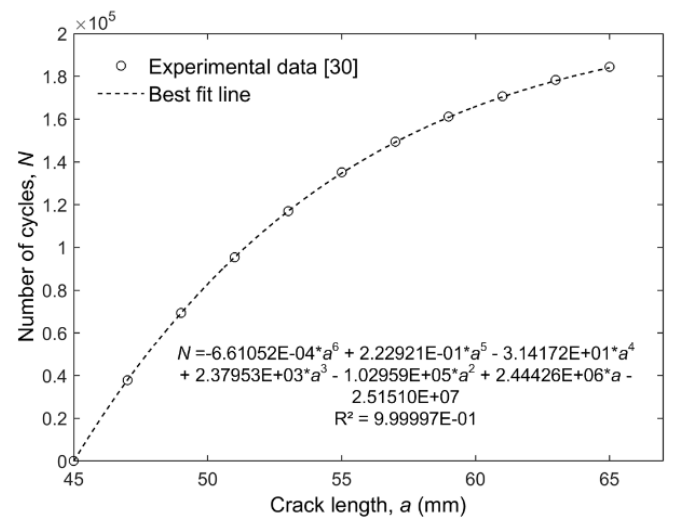

Fig. 5. Experimental data and the polynomial fit.

The numerically estimated fatigue life data using different $\Delta K_{e q}$ models are plotted in Fig. 6. The polynomial fit of the experimental fatigue life is also plotted in Fig. 6 for comparison. For better understanding, the close-up views of Fig. 6 near the origin and at the end of the graph is shown in Fig. 7. The percentage relative error in the predicted fatigue life is calculated using the fitted data as reference and is plotted in Fig. 8. In the present numerical investigation, the percentage relative error is calculated by using the fitted polynomial as reference. Fig. 5 also shows the goodness of fit.

It can be seen from Fig. 8 that at the initial stages all the $\Delta K_{e q}$ models presented in Table 1 shows significant deviation from the reference values. It can be noted that at higher crack increments, the percentage relative error is relatively less. The predicted fatigue life model using Irwin's model [5] is closer to the reference fatigue life from the experiment. The fatigue life estimate using model proposed by Hussain et al. [7] provides next closest solution. Compared to Irwin's [5] and Hussain et

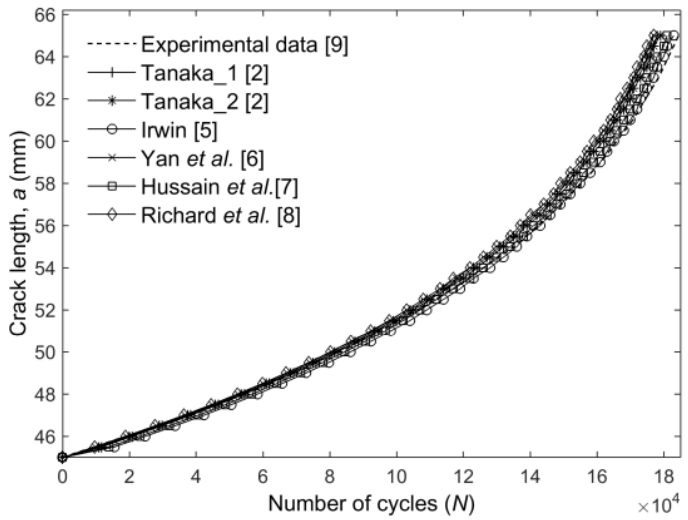

Fig. 6. Experimental and simulated fatigue life.

al.'s [7] models, the other methods offers more conservative fatigue life predictions. Yan et al. [6] model and Richard et al. [8] model are more conservative. Also, it is observed from the similar numerical investigations that when the mode mixity is less, most of the models predicting mixed mode fatigue life close to experimental fatigue life. When the mode mixity is high, the fatigue life prediction using some of the $\Delta K_{e q}$ models are higher compared to Irwin's [5] and Hussain et al.'s [7] models. A detailed investigation of the present study using a variety of benchmark examples along with some recommendation for the reduction of initial errors is available in $[11,12]$.

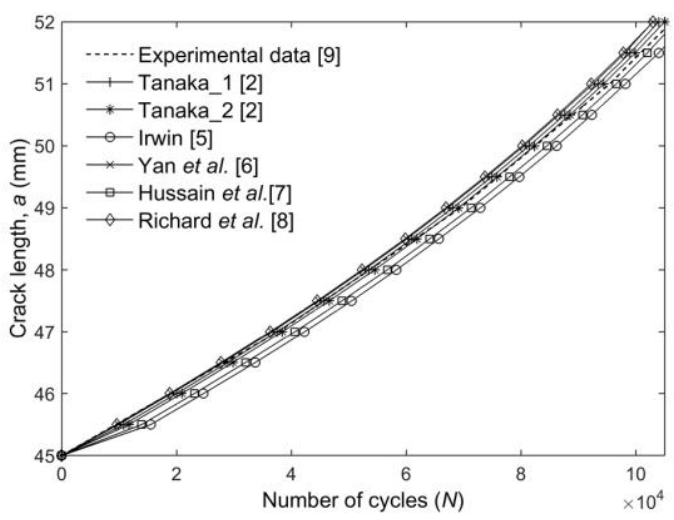

(a)

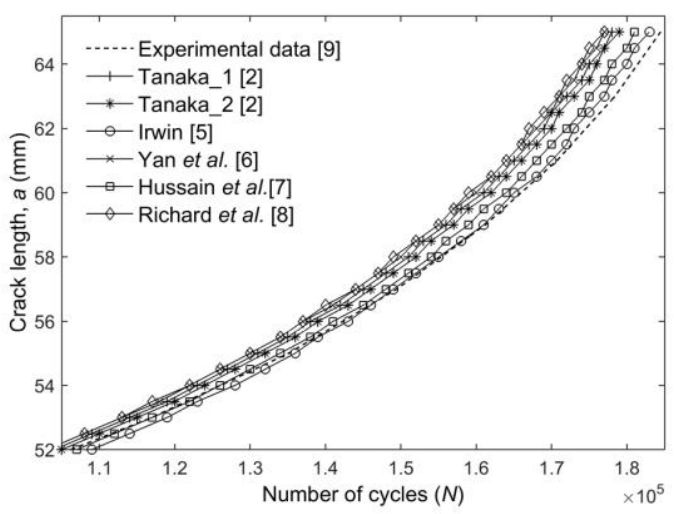

(b)

Fig. 7. (a) Close-up view of Fig. 6 near the origin and (b) at the end of the graph 


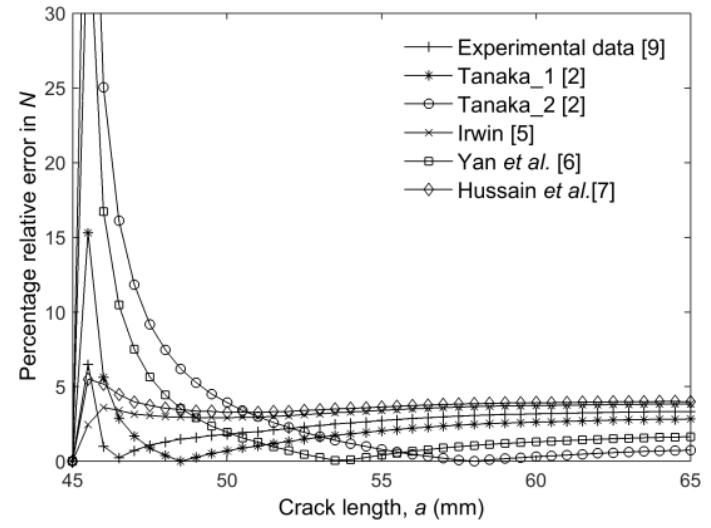

Fig. 8. Relative percentage error in the estimated fatigue life using different equivalent SIF models.

\section{Conclusions}

Performance analysis of various $\Delta K_{e q}$ models in predicting the fatigue life is carried out in this study. The present study is conducted using the limited available experimental results; the present study provides certain useful observations. The results of the present investigation indicate that fatigue life predicted using the $\Delta K_{e q}$ models based on Irwin [5] and Hussain et al. [7] models are closer to the experimental fatigue life used in the present numerical investigation. Among the above two, fatigue life prediction using Irwin's [5] model is more close to the experimental fatigue life. One of the models proposed by Tanaka [2] is also predicting fatigue crack growth life close to the experimental data. The remaining three models unsed for the analysis provides more conservative solution, among them the model proposed by Richard et al. [8] is the most conservative model. It is observed in the present investigation that the when the mode mixity is less, most of the models are predicting fatigue life close to the experimental results. However, when there exists a higher mode mixity, there is a significant deviation among the fatigue life prediction by different models and the models proposed by Irwin [5], and Hussain et al. [7] are found to be performing well. The present investigation offers some useful insights into the prediction of mixed mode fatigue crack growth life, which is essential from the damage tolerance point of view.

The authors are thankful to Cornell Fracture Group, Cornell University, New York for allowing to use the FRANC2D software.

\section{References}

1. A. Paris, F. Erdogan, J. Basic Eng. 85, 528-533 (1963).

2. K. Tanaka, Eng. Fract. Mech. 6, 493-507 (1974).

3. A.B. Patel, R.K. Pandey, Fatigue Fract. Eng. Mater. Struct. 4, 65-77 (1981).
4. F. Erdogan, G.C. Sih J. Basic. Eng. 85, 519-525 (1963).

5. G. Irwin, J. Appl. Mech. 24, 361-364 (1957).

6. Y. Xiangqiao, D. Shanyi, Z. Zehua, J. Appl. Mech. 43, 471-475 (1992).

7. M. Hussain, S. Pu, J. Underwood, Fract. Anal. ASTM STP 560, 2-28 (1974).

8. H.A. Richard, F.G. Buchholz, G. Kullmer, M. Schöllmann, Key Eng. Mater. 251, 251-260 (2003).

9. S. Ma, X.B. Zhang, N. Recho, J. Li Int. J. Fatigue 28, 1780-1790 (2006)

10. H.A. Richard, K. Benitz, Int. J. Fract. 22, 55-58 (1983).

11. S. Sajith, K.S.R.K. Murthy, P.S. Robi, J. Inst. Eng. India Ser. C (2017)

12. S. Sajith, K.S.R.K. Murthy, P.S. Robi, AIP Conf. Proc. 1943, (2018) 